\title{
The DLR RailwayDynamics Library: the Crosswind Stability Problem
}

\author{
Andreas Heckmann ${ }^{1}$ Gustav Grether ${ }^{2}$ \\ ${ }^{1,2}$ Institute of System Dynamics and Control, German Aerospace Center (DLR), Germany, \\ andreas.heckmann@dlr.de
}

\begin{abstract}
High crosswinds affect the stability of railway vehicles, in particular if they run on very high speed to reduce traveling time, if they are configured as double-deck cars to increase the number of passenger seats and if they use lightweight design in order to reduce life-cycle costs. This is why crosswind stability is an active field of research within the project Next Generation Train. However, this field relies on the cooperation of two different domains, namely aerodynamics and vehicle dynamics. With this background a crosswind stability tool was implemented in Modelica as a part of the DLR RailwayDynamics Library. This tool gathers data from scaled wind tunnel measurements and multibody data on the railway vehicle in order to rapidly analyze and assess the risk of overturning due to high crosswinds. To a large extent the tool is oriented towards the associated homologation rules and standards. However, the tool is as well supposed to support future advancements of these standards by providing capabilities for the stochastic analysis of the crosswind stability problem.

vehicle dynamics, aerodynamics, railway vehicles, crosswind stability, aerodynamic admittance, stochastic analysis
\end{abstract}

\section{Introduction}

\subsection{Motivation}

Crosswind stability addresses the risk, that vehicles running on high speed are prone for overturning, if high crosswinds occur. At DLR, it is a particular subject of research, since the long-term internal project Next Generation Train (NGT) copes with three key features that aggravate the problem: it is a very high-speed train in double deck configuration and light-weight design. Nevertheless, this train concept has been proposed since it facilitates objectives such as low energy consumption and low life-cycle costs per passenger even for reduced traveling times.

From the technical point of view, crosswind stability is a multidisciplinary issue, since aspects of aero- and vehicle dynamics have to be taken into account. This is why the homologation rules, specified by regulation of the European Commission (TSI HS RST 2008) and the associated standard (EN 14067-6: 2010) address both: scaled wind tunnel experiments in order to characterize the aero- dynamic properties and multibody simulations to study the mechanical behavior of the railway vehicle under consideration.

With this background, a subpackage of the DLR RailwayDynamics Library (Heckmann et al., 2014a), (Schwarz et al., 2015) has been implemented in Modelica which aims at the assessment of railway vehicles with respect to crosswind stability. To a large extent, the tool is oriented towards (EN 14067-6: 2010) and the simulation procedures defined there including a non-normative but promising stochastic approach in Appendix J. But beyond that, the implementation is intended to support various research activities in aerodynamics and vehicle dynamics control within the NGT project, see e.g. (Fey et al., 2014), (Heckmann et al., 2014b).

\subsection{Overview on Vehicle Assessment}

The basic assessment scenario is defined in (EN 14067-6: 2010) as follows: At given train speed, wind velocity and wind direction, aerodynamic forces and torques are applied to a multibody train model. These applied forces and torques lead to an unloading of the wheels at the windward side of the train. This unloading is interpreted to indicate the risk of overturning.

In detail, a major assessment issue is the so-called critical wind speed that is defined as the wind velocity, at which $90 \%$ wheel unloading compared to the static load occurs. In general, the evaluation procedure requires to iteratively vary the wind velocity as an input parameter till the multibody simulation results show that the remaining wheel load is $10 \%$ of the static load.

The critical wind speed is evaluated for several train velocities, these sample points are then connected to construct a curve called the Characteristic Wind Curve (CWC). In order to meet the homologation criterion the CWC of the considered vehicle must completely run above the Characteristic Reference Wind Curve (CRWC) defined by (TSI HS RST 2008). As illustrative examples, Fig. 1 presents the CWC of the NGT train head which meets the homologation criteron, while the initial NGT coach design does not. 


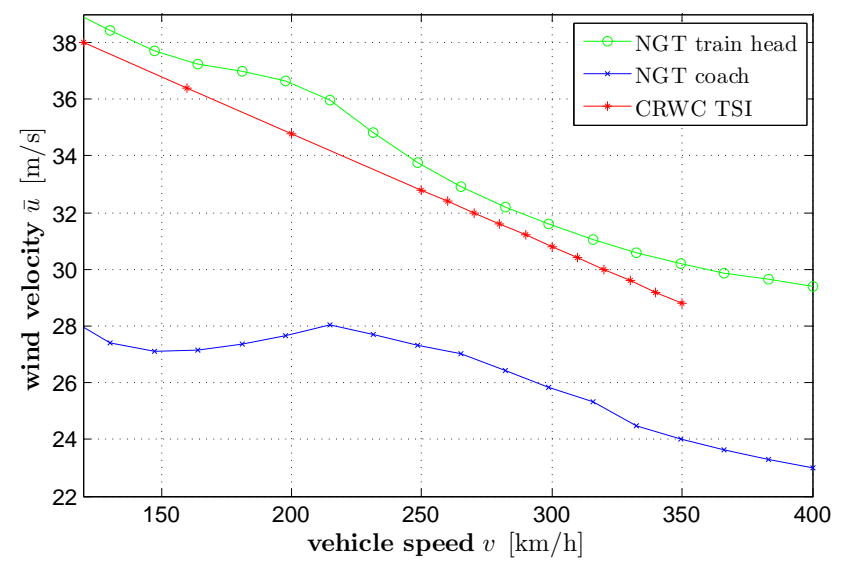

Figure 1. Characteristic Wind Curve (CWC) of the NGT train head and the NGT coach compared to the reference curve CRWC from (TSI HS RST 2008), cf. (Heckmann et al., 2014b).

\section{Aerodynamic Loads}

\subsection{Fundamentals}

The above described assessment scenario requires to specify loads, i.e. the aerodynamic forces and torques that are to be applied to the mechanical train model. These loads are commonly expressed by means of aerodynamic coefficients $c_{i}=c_{i}(\beta)$ and $c_{j}=c_{j}(\beta)$ as function of the yaw angle $\beta$ as follows (Baker et al., 2009):

$$
\begin{aligned}
\bar{f}_{i} & =\frac{1}{2} \rho A c_{i}(\beta) \bar{V}^{2} \\
\bar{m}_{j} & =\frac{1}{2} \rho A h c_{j}(\beta) \bar{V}^{2} .
\end{aligned}
$$

In (1) and (2), $\bar{f}_{i}$ and $\bar{m}_{j}$ represent the vector components of force and torque, $\rho$ is the density of the air, $A$ and $h$ the reference area and height, respectively. $\bar{V}$ denotes the wind speed relative to the vehicle, which follows from vectorial decomposition considering the vehicle speed $v$, the wind velocity $\bar{u}$ and the angle $\beta_{w}$ between track and wind, see Fig. 2.

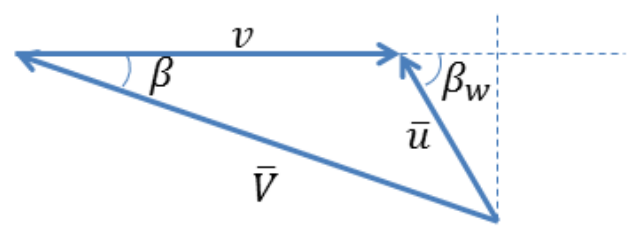

Figure 2. Vector decomposition to evaluate the wind speed relative to the vehicle $\bar{V}$.

It is state-of-the art to identify aerodynamic coefficients in scaled wind tunnel measurements as visualized in Fig. 3 and normalize the results with $A=10 \mathrm{~m}^{2}$ and $h=3 \mathrm{~m}$ according to (TSI HS RST 2008). Note that the (EN 140676: 2010) also approves numerical CFD simulation under certain conditions.

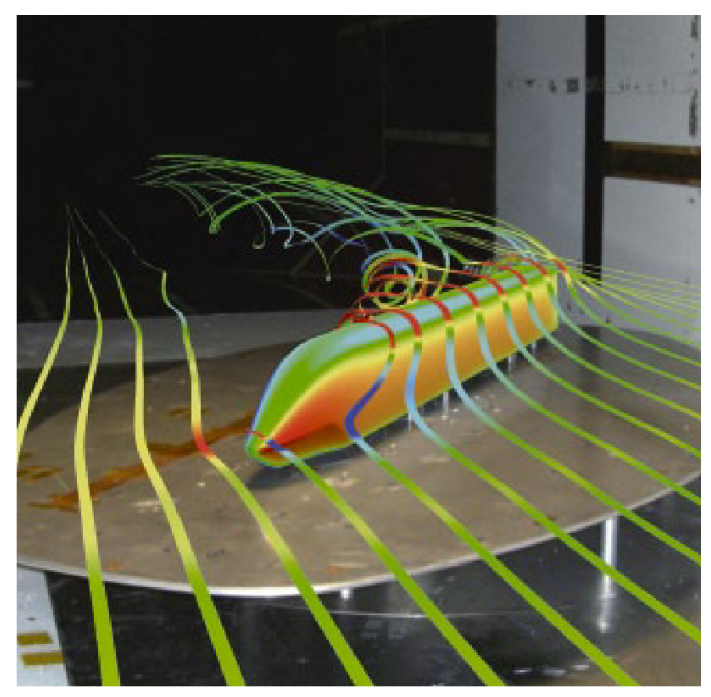

Figure 3. Calculated streamlines and pressure distribution on the NGT model scale 1:25 in the Cologne Cryogenic Wind Tunnel under cross wind conditions, see (Heckmann et al., 2014b).

Eq. (1) and (2) define the aerodynamic loads as a function of $\bar{V}$ which in turn depends on the wind velocity $\bar{u}$ according to Fig. 2 or more general, on the underlying wind gust model. Three different approaches to represent the wind gust are implemented in the Modelica RailwayDynamcis Library:

1. Steady approach: $\bar{u}=$ const

2. Quasi-steady approach: Eq. (1) and (2) are assumed to be valid even if the wind velocity changes in time, i.e. $\bar{u}=u(t)$. In detail, (EN 14067-6: 2010) defines the so-called Chinese Hat gust model in Fig. 4 which specifies a wind field fixed along the track at which the vehicle runs at constant speed $v$. For (1) and (2), $\bar{u}$ then follows from $\bar{u}=\bar{u}(s)=\bar{u}(v \cdot t)$,

3. Unsteady approach: The mean wind forces $\bar{f}_{i}$ and torques $\bar{m}_{j}$ are superimposed with fluctuating parts $f_{i}^{\prime}$ and torques $m_{j}^{\prime}$ (Baker, 1991), i.e.

$$
f_{i}=\bar{f}_{i}+f_{i}^{\prime}, \quad m_{j}=\bar{m}_{j}+m_{j}^{\prime},
$$

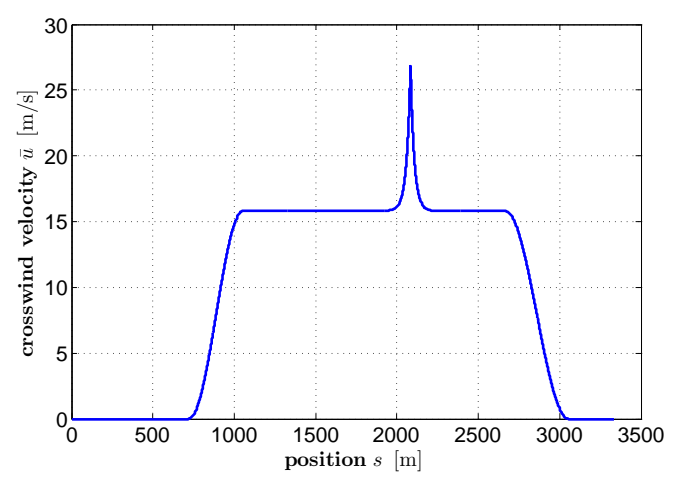

Figure 4. Crosswind velocity definition "Chinese Hat" according to (EN 14067-6: 2010). 


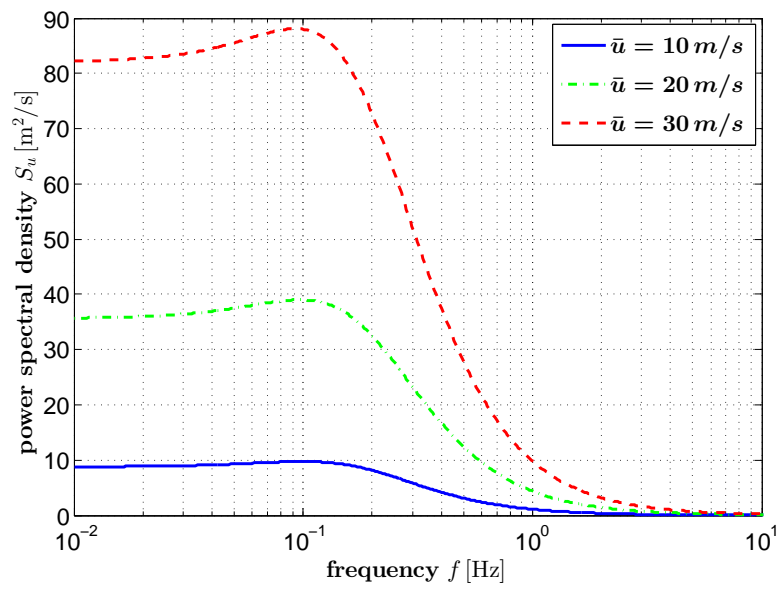

Figure 5. Power spectral density example of the turbulent wind according to (Cooper, 1984) for three different wind velocities $\bar{u}$, train speed $v=400 \mathrm{~km} / \mathrm{h}$ and wind angle $\beta_{w}=90^{\circ}$.

which are specified in the frequency domain as described in the next section.

\subsection{Unsteady Aerodynamics}

Unsteady wind may be approximated as a Gaussian stochastic process and therefore may be characterized by the power spectral density (PSD). Cooper derived the following wind spectrum relative to the moving vehicle $S_{u}$ as function of the frequency $f$ (Cooper, 1984):

$$
S_{u}=\frac{4 \tilde{f}}{f} \frac{\sigma_{u}^{2}}{\left[1+70.8 \tilde{f}^{2}\right]^{\frac{5}{6}}}\left[c_{u}+\left(1-c_{u}\right) \frac{0.5+94.4 \tilde{f}^{2}}{1+70.8 \tilde{f}^{2}}\right]
$$

that include the following definitions and parameter values:

- the coefficient $c_{u}: c_{u}=\left(\frac{u}{\bar{V}} \cos \beta_{w}+\frac{\bar{u}}{\bar{V}}\right)^{2}$.

- the root mean square of the wind velocity fluctuations $\sigma_{u}: \sigma_{u}=0.245 \cdot \bar{u}$, here inserted according to (EN 14067-6: 2010).

- the turbulence length scale in wind direction ${ }^{x} \mathrm{Lu}$ : ${ }^{x} L u=96.0395 \mathrm{~m}$, see (EN 14067-6: 2010).

- the compound length scale $L_{u}$ : $L_{u}={ }^{x} L u \sqrt{c_{u}+0.706\left(1-c_{u}\right)}$

- the normalized frequency $\tilde{f}: \tilde{f}=f \frac{L_{u}}{\bar{V}}$

Fig. 5 shows exemplary spectra in order to illustrate (4).

In order to generate a representation of $S_{u}$ in time domain, the fluctuation wind velocity $u^{\prime}$ may be evaluated by superimposing $n$ discretized frequencies $f_{i}$ with amplitude $A_{i}=A_{i}\left(f_{i}\right)$ and random phase $\phi_{i}$ :

$$
u^{\prime}=\sum_{i=1}^{n} A_{i} \sin \left(2 \pi f_{i} \cdot t+\phi_{i}\right), A_{i}=\sqrt{2 S_{u, i}\left(f_{i+1}-f_{i}\right)} .
$$

In reality, it has been observed that force fluctuations don't follow wind velocity fluctuations without attenuation or

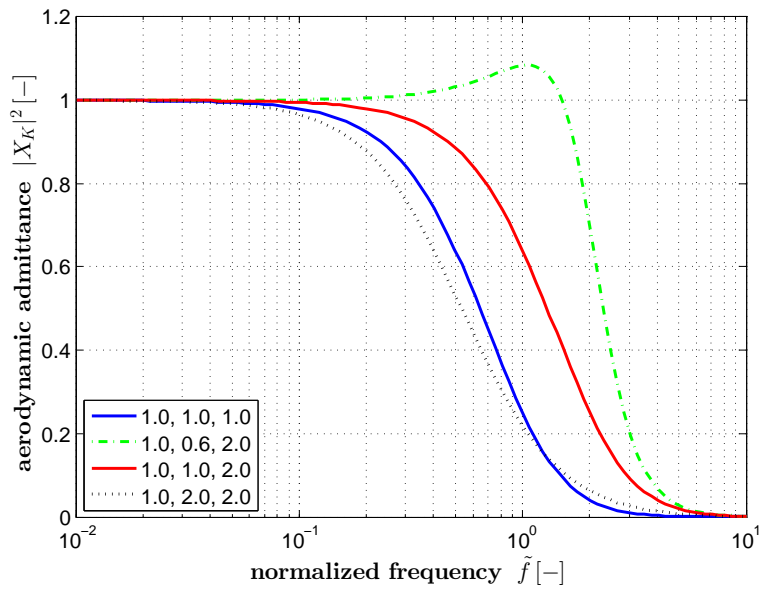

Figure 6. Aerodynamic addittance function for various parameter triples $\hat{k}, \hat{\xi}, \hat{f}$.

lag, so that an additional dynamics transfer behavior has to be considered by the aerodynamic admittance function $\left|X_{K}(f)\right|^{2}$ for each of the six force or torque components $K$ and their associated spectra $S_{K}$ :

$$
\left|X_{K}(f)\right|^{2}:=\frac{1}{\left(\rho A c_{k} \bar{u}\right)^{2}} \frac{S_{K}}{S_{u}}, \quad k=i, j, \quad K=f_{i}^{\prime}, m_{j}^{\prime} .
$$

The measurement of the aerodynamic admittance for high-sped trains is a field of active research, which the RailwayDynamics Library is supposed to support. As an initial approach, it is refered to the following model, which uses three free, dimensionless parameters $\hat{k}, \hat{f}$ and $\hat{\xi}$, which are fitted to approximate wind tunnel measurements as good as possible (Sterling et al., 2009):

$$
\left|X_{K}(f)\right|^{2}:=\frac{1}{\hat{k}\left(\left[1-\left(\frac{\tilde{f}}{\hat{f}}\right)^{2}\right]^{2}+\left[2 \hat{\xi} \frac{\tilde{f}}{\hat{f}}\right]^{2}\right)^{2}}
$$

Fig. 6 gives an impression on parameter values from literature specifying the aerodynamic admittance.

\section{Vehicle Dynamics}

The (EN 14067-6: 2010) refers to three vehicle models with increasing complexity:

- The 2D three-mass model, which is not implemented in the RailwayDynamics Lib.

- The five-mass model without wheel-rail contact which is supposed to be used in a steady-state scenario.

- The multibody model with wheel-rail contact which is intended to be utilized for transient simulation tasks, in which quasi-steady or unsteady aerodynamic loads are applied. 


\subsection{The Simplified Five-Mass Model}

Fig.7 presents the structure of the five-mass model, as it is defined in (EN 14067-6: 2010, Appendix H) It considers two unsprung bodies which are the wheelsets of the railway vehicle, two primary suspended bodies representing the bogie frames including all attachments and the car body, which is connected to the bogies via secondary suspensions.

The four wheel-rail forces $Q_{i j}, i, j=1,2$, in Fig.7 are interpreted as supporting or reaction forces due to the condition that the wheels do no lift off. Note, that at most $90 \%$ wheel unloading is permitted which in turn defines that lift-off or loss of contact at the wheel-rail interface is not admissable and motivates to disregard the wheel-rail contact for the sake of simplicity.

The model takes all together 11 degrees of freedom into account. These are the lateral, vertical and roll motion of bogies and the car body, which may in addition rotate around its pitch and yaw axis. The vertical and lateral spring elements of the primary and secondary suspensions are equipped with bump stops specified by a non-linear stiffness characteristic as well proposed in (EN 14067-6: 2010, Appendix H). The rotational stiffness of an anti-roll bar as part of the secondary suspensions is supposed to reduce the tilting of the carbody due to wind torques acting around the longitudinal or $\mathrm{x}$-axis.

Since this model is used in a steady-state scenario, transient behavior or modeling of damping devices actually is irrelevant but nevertheless is introduced in parallel to all spring elements. Due to the bump stops, the model is nonlinear and the analysis is organized as a time simulation that is intended to converge against its final and steady state as a result of the applied constant wind loads.

In summary, the five-mass model is supposed to facilitate a simple analysis to be feasible in early engineering phases yielding conservative results with comparable low critical wind speeds. To this aim, the suspension elements are not modeled considering design details but are represented by a set of generalized stiffness parameters and

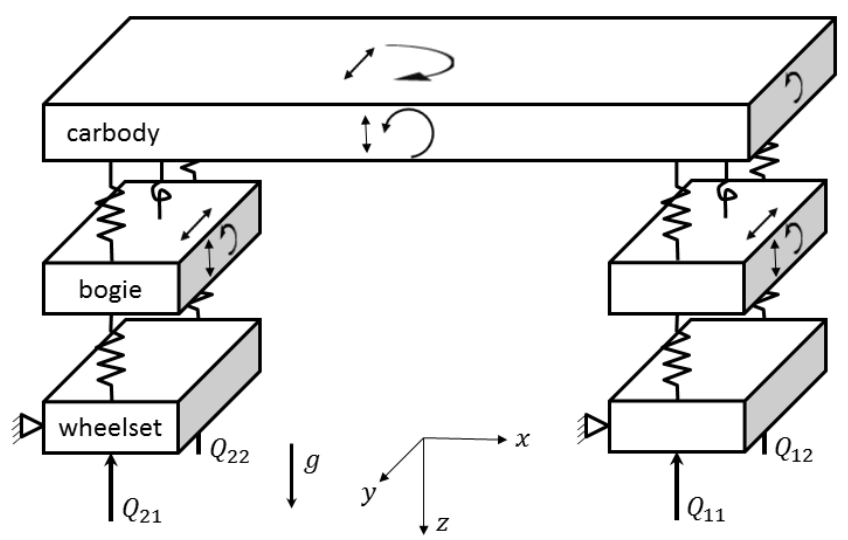

Figure 7. Structure of the simplified five-mass model according to (EN 14067-6: 2010).

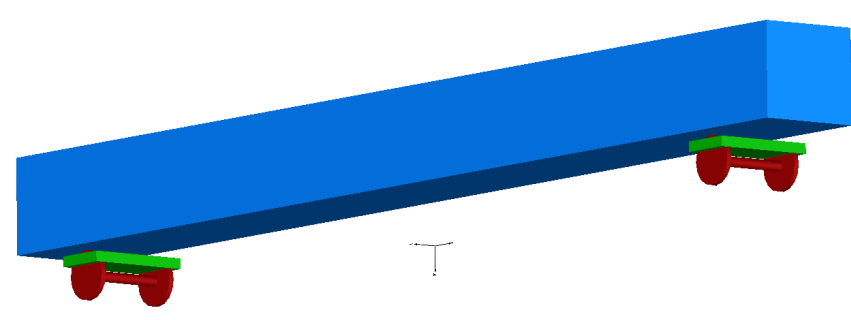

Figure 8. Animation of the five-mass-model.

very essential geometric information. Fig. 8 shows an animation of the five-mass model in Modelica.

\subsection{The Multibody Model}

The multibody model to described here is tailored to the lightweight intermediate car of NGT project, but may easily adapted to conventional high-speed railway vehicles. The most prominent up-grade to the five-mass model concerns the non-linear wheel-rail contact that is considered on basis of the geometry of the standarized UIC 60 rail and the WS 1002 profile geometry.

To this aim, the Modelica RailwayDynamics Library (Heckmann et al., 2014a) employs the distance $\Delta=$ $\Delta(s, y, \varphi, \psi)$ as a function of the wheel profile coordinate $s$, $\underline{s} \leq s \leq \bar{s}$, and the lateral displacement, roll and yaw angle between wheel and rail, see Fig. 9. The contact position $s^{*}$ defined as a weighted mean value of $s$ using the regularization parameter $\alpha$ is a continuous function of $y, \varphi$ and $\psi$ and thus constitutes a smooth contact formulation, see (Arnold and Netter, 1997):

$$
s^{*}:=\frac{\int_{\underline{s}}^{\bar{s}} s e^{\frac{\Delta}{\alpha}} \mathrm{d} s}{\int_{\underline{s}}^{\bar{s}} e^{\frac{\Delta}{\alpha}} \mathrm{d} s} .
$$

However for transient analysis, the (EN 14067-6: 2010) allows to monitor the wheel-rail forces utilizing a $2 \mathrm{~Hz}$ low-pass filter to evaluate the wheel unloading criterion. Therefore, it cannot be completely ruled out that loss of contact occurs and is admissible for very short time periods. In addition, overloading and in turn wheel lift-off

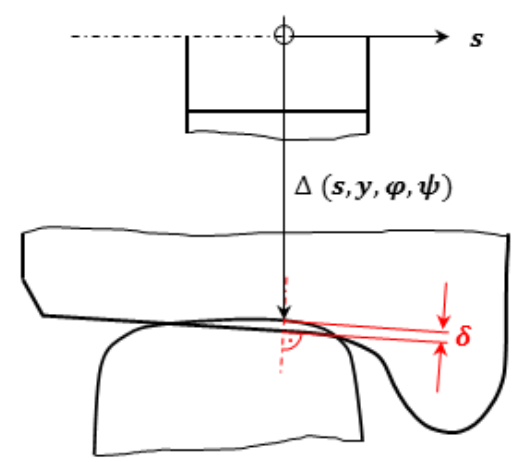

Figure 9. Sketch to illustrate the wheel-rail contact quantities (exaggerated presentation of the penetration $\delta$ ). 


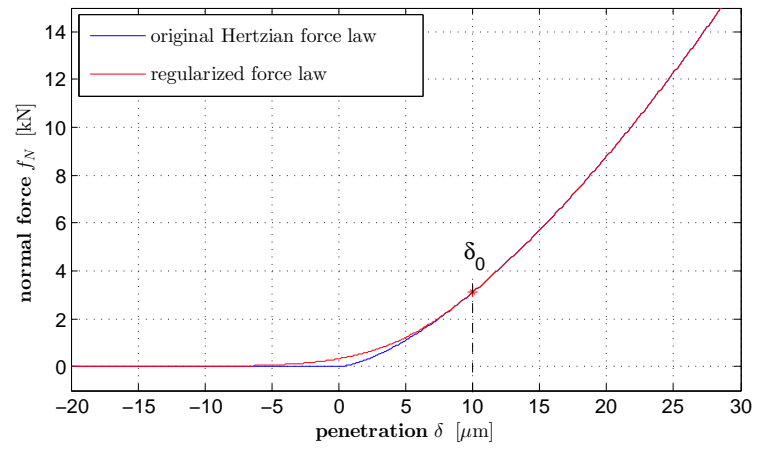

Figure 10. Illustration of the regularized contact force law.

may occur during the iteration process to determine the critical wind speed.

In order to take this into account, the wheel-rail contact algorithm of the Modelica RailwayDynamics Library had to be adapted. In particular the kinematic constraint (Heckmann et al., 2014a, (6)) is replaced by a regularized penalty contact formulation, in which the penetration $\delta$ of the wheel and the rail body in Fig. 9 features a non-linear spring element to evaluate the normal contact force $f_{N}$ according to the Hertzian theory (Hertz, 1882):

$$
f_{N}= \begin{cases}\delta<\delta_{0}: & a \cdot e^{-b(c+\delta)^{2}}, \\ \delta \geq \delta_{0}: & c_{H} \cdot \delta^{\frac{3}{2}},\end{cases}
$$

where $\delta_{0}>0, a:=c_{H} e^{\frac{3}{4}} \delta_{0}^{\frac{3}{2}}, b:=\frac{3}{4 \delta_{0}^{2}}, c:=-2 \delta_{0}$.

The coefficient $c_{H}$ is a function of the material properties and the local curvatures of the contact partners at the point of contact, while $a, b$ and $c$ are defined in such a way that $f_{N}$ is two times continuously differentiable for all values of $\delta$ and in particular for $\delta=\delta_{0}$, see Fig. 10. The proposed regularization prevents chattering in the vicinity of wheel lift-off situations and therefore improves the numerical robustness. In addition, this elastic contact, to be distinguished to the quasi-elastic formulation in (Heckmann et al., 2014a), requires damping to be numerical feasible.

It is state of the art in multibody analysis of railway vehicles to take the elastic compliance of the track into account that is excited by large wheel-rail forces. This is in particular important if these forces are a significant result of the analysis. Therefore, the track superstructure shown in Fig. 11 is presented as one body with three sprung and damped degrees of freedom, which is assumed to follow each wheel pair as a so-called moving track model, e.g. see (Iwnicki, 2006, Ch. 12)

The NGT running gears use so-called independently rotating wheel sets (IRW set) with two wheels attached to a wheel carrier. Active guidance control (Kurzeck et al., 2014) provides running stability and low wear properties of this configuration which is a main objective of the NGT project.

In order to be consistent, these model upgrades implicate a more complex motion composition compared to

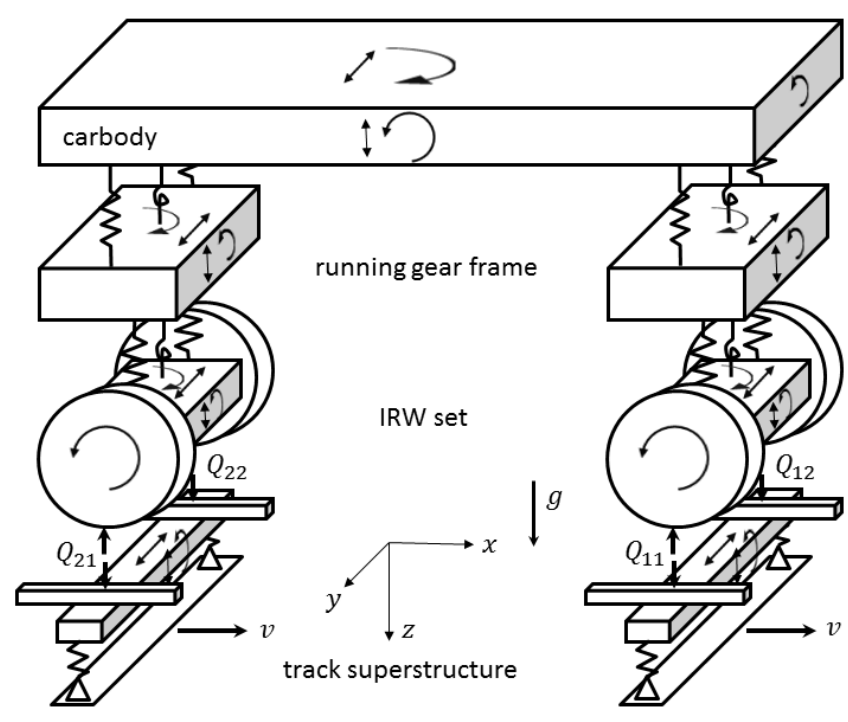

Figure 11. Structure of the multibody model of the NGT intermediate car (all springs include dampers connected in parallel).

Sec. 3.1 that requires the consideration of all together 31 degrees of freedom which are indicated in Fig. 11. Apart from that, the multibody model, whose animation is shown in Fig. 12, sticks to the concept of the five-mass model to present all suspension elements by a set of generalized stiffness and damping parameters and very essential geometric information. This modeling idea is well suited to be used in early engineering phases, when detailed information on the design of the suspensions are not yet available and therefore fits to the scope of the RailwayDynamics Library.

\subsection{Negotiating Curves}

For comfort reason, the maximum lateral acceleration that passengers are supposed to experience while the vehicle is negotiating curves is restricted to $1 \mathrm{~m} / \mathrm{s}^{2}$. Therefore, the lay-out of railway tracks also includes superelevations and the maximum speed at which the vehicles runs through the curve is limited as a function of curve superelevation and radius in order to meet this requirement.

This property is exploited by the (EN 14067-6: 2010) to

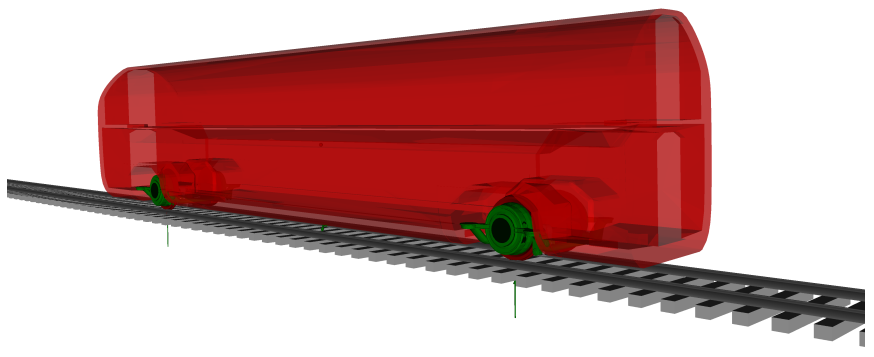

Figure 12. Animation of the NGT intermediate car multibody model. 


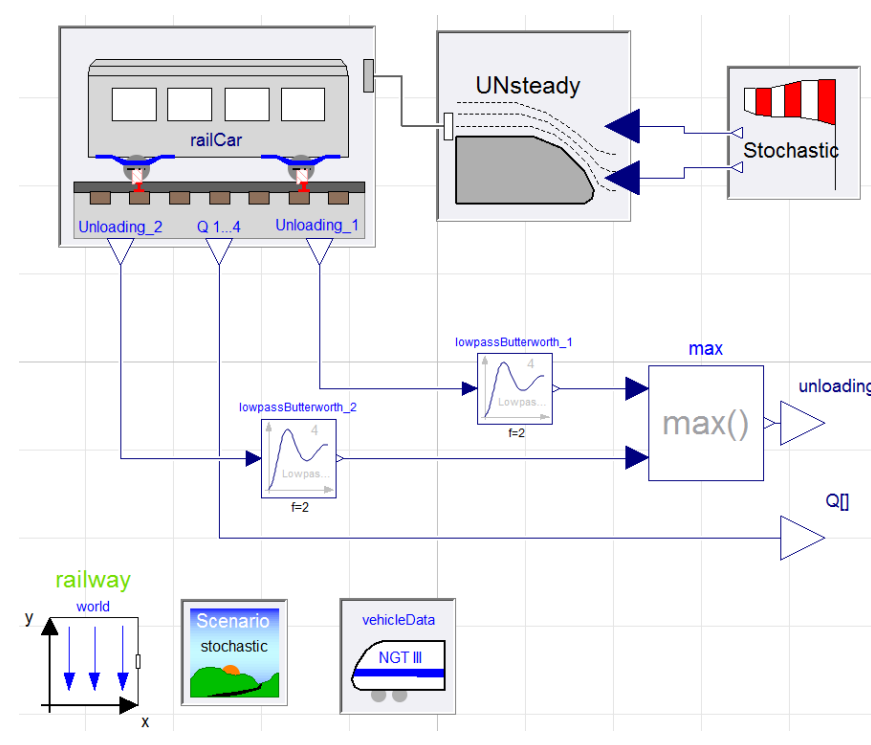

Figure 13. Diagram layer of a crosswind stability model of the Modelica RailwayDynamics Library.

address the crosswind stability while negotiating curves. The evaluation of the CWCs are additionally parametrized with the so-called unbalanced lateral acceleration $a_{q}$, which can take values between -1 and $1\left[\mathrm{~m} / \mathrm{s}^{2}\right]$, i.e. $-1 \mathrm{~m} / \mathrm{s}^{2} \leq a_{q} \leq 1 \mathrm{~m} / \mathrm{s}^{2}$. The introduction of this parameter into the Modelica crosswind scenario is straight forward by specifying the direction and value of the gravity vector accordingly.

\section{Implementation}

Fig. 13 gives an overview on the structure of a crosswind stability model of the Modelica RailwayDynamics Library. Vehicle data are separated from the model instances and organized by data records, which in turn are substructured in aerodynamical and mechanical information. Another record called Scenario organises information to perform the specific simulation task, see Fig. 14.

Wind generation, aerodynamical load evaluation and vehicle running dynamics are separated in three model components, so that it is easy to exchanged e.g. the stochastic wind with a chinese hat wind gust instance or subsitute steady for unsteady aerodynamic approach.

In order to facilitate robust initialization, the application of the aerodynamic loads $f_{i}$ and $m_{j}$ from (3) is delayed in time using a first order low pass filter with time constant $t_{0}$, i.e.:

$$
\breve{f}_{i}=\left(1-e^{-\frac{t}{t_{0}}}\right) f_{i}, \quad \breve{m}_{j}=\left(1-e^{-\frac{t}{t_{0}}}\right) m_{j} .
$$

The railCar instance in Fig. 13 provides the wheelunloading of each running gear as a function of time, which then is low-pass filtered according to (EN 140676: 2010). The output of the $\max ()$ block evaluates the final simulation result or the crosswind stability criterion, respectively.

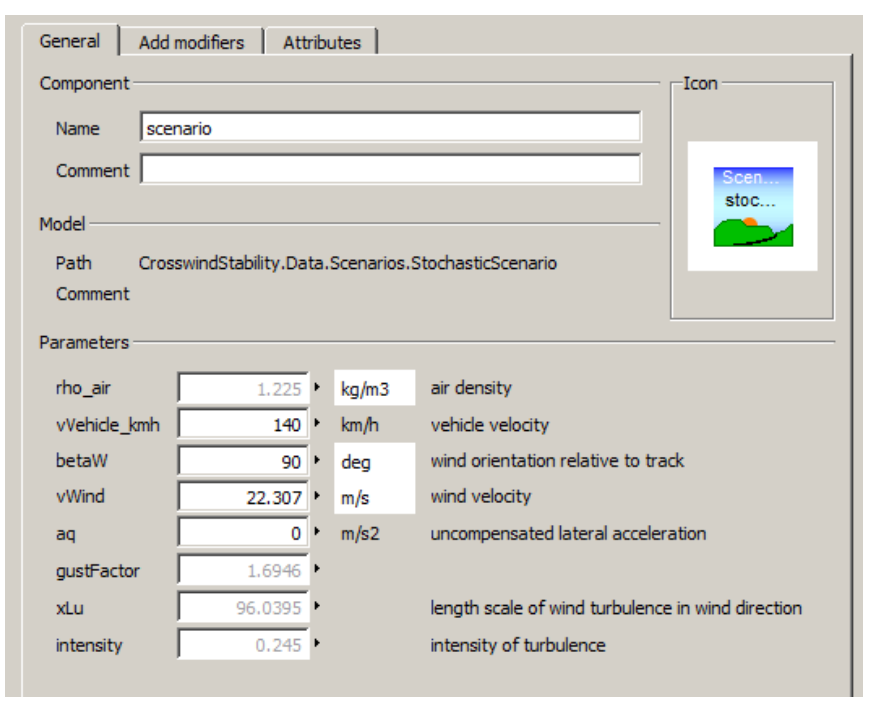

Figure 14. Parameter menu of the scenario data record.

In order to determine the critical wind speed, a function find_Vcwc is defined, which iteratively simulates the crosswind stability model, while the wind speed is varied systematically. The function terminates and returns the critical wind speed, if the model simuation results show 90\% wheel unloading:

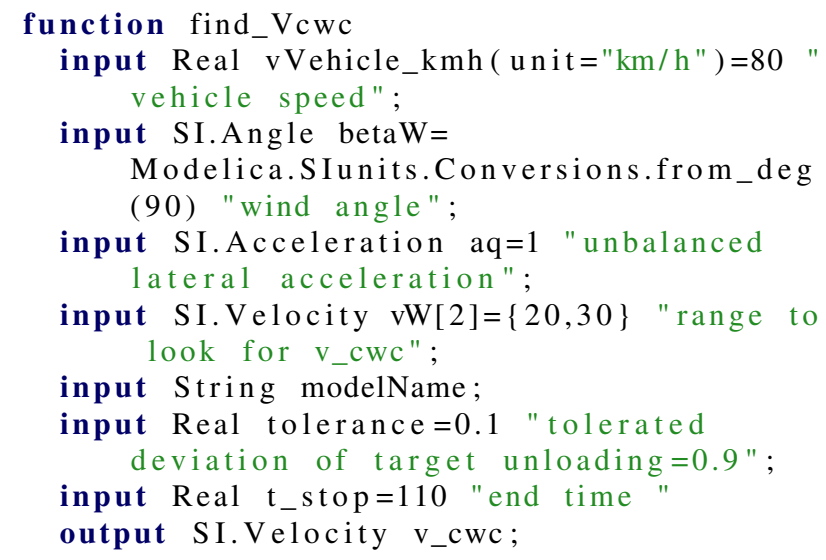

A second function plot_CWC not only evaluates one critical wind speed, but provides a plot of the critical wind curve as shown in Fig. 1.

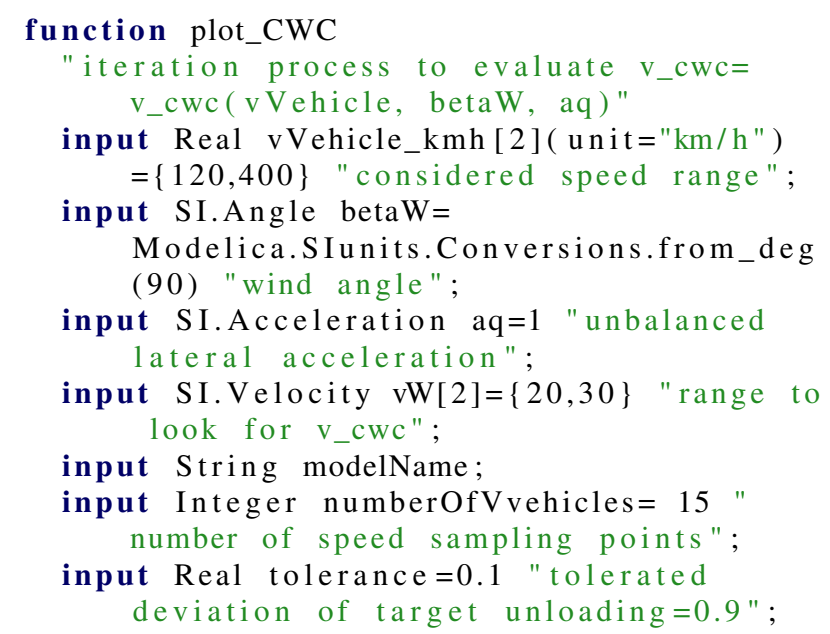


input Real t_stop=110 "end time"

output SI.Velocity v_cwc [

numberOfVvehicles ];

\section{Exemplary Results}

\subsection{The Five-Mass Model}

The (EN 14067-6: 2010) offers the opportunity to verify the implementation of the steady scenario with the fivemass model, since its Appendix $\mathrm{H}$ contains the input data and the results of two example vehicles. Fig. 15 presents a comparison of Modelica RailwayDynamcis Library results with the CWC from the EN standard for Vehicle 2 and two unbalanced accelerations. The large correspondence of results can be stated, a slight derivation only occurs for $v=80 \mathrm{~km} / \mathrm{h}$ vehicle speed, which is to be further investigated.

The CWC of the NGT in Fig. 1, which has been introduced in order to give an overview on the vehicle assessment methodology in Sec. 1.2, has also been generated using the five-mass model, see also (Heckmann et al., 2014b) for a more detailed discussion.

\subsection{The Multibody Model of the NGT Coach}

With the exception of Fig. 19, all results to be given in this section have been obtained using the scenario shown in Fig. 14, which will turn out to lead to $90 \%$ wheel unloading. The associated transient wind velocities are plotted in Fig. 16.

The parameters to be used in (6) are intended to be gained in wind tunnel measurements, which are not yet available. Therefore, hypothetical addmittance parameters have been introduced in order evaluate preliminary and exemplary results. Following a proposal of (Baker, 2010), the values below have been chosen for the most important force and torque components:

$$
\begin{array}{llll}
\text { side force: } & \hat{k}=1, & \hat{\xi}=1, & \hat{f}=2.0 \cdot \sin (\beta), \\
\text { lift force: } & \hat{k}=1, & \hat{\xi}=1, & \hat{f}=2.5 \cdot \sin (\beta), \\
\text { roll moment: } & \hat{k}=1, & \hat{\xi}=1, & \hat{f}=2.0 \cdot \sin (\beta) .
\end{array}
$$

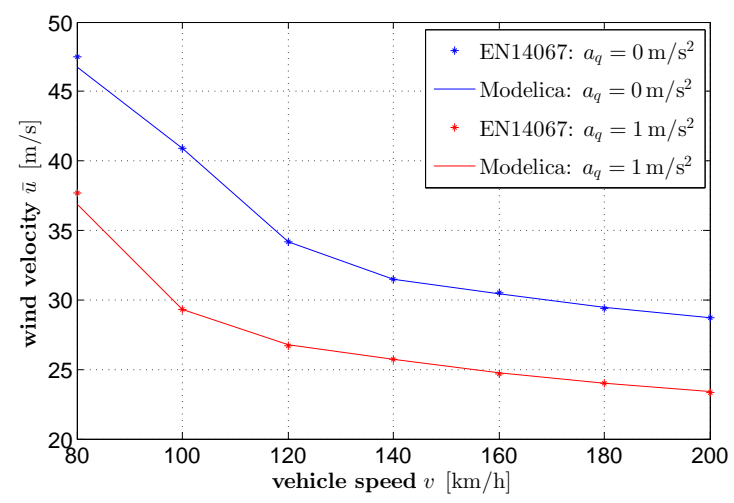

Figure 15. Comparison of the CWC evaluated in Modelica with (EN 14067-6: 2010), Vehicle 2 in Appendix H.

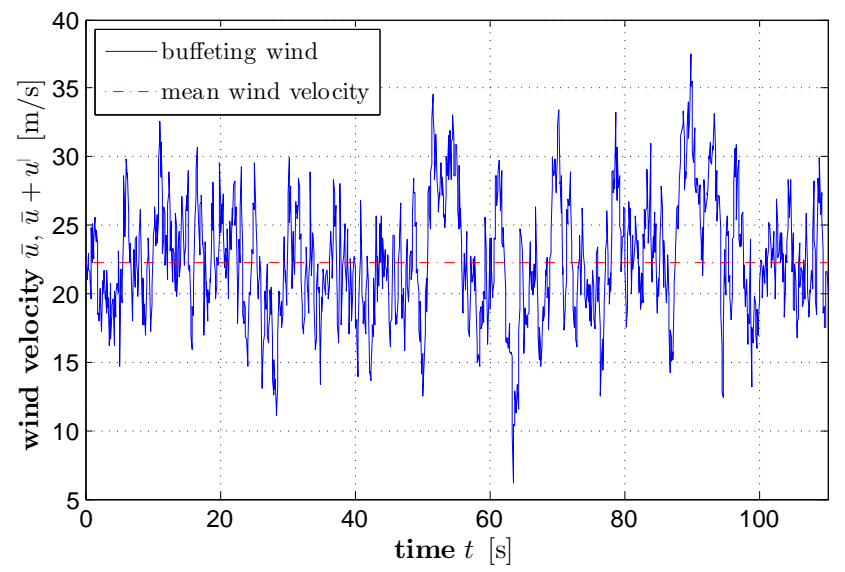

Figure 16. Transient velocities of buffeting wind $(\bar{u}=$ $22.307 \mathrm{~m} / \mathrm{s}, \sigma_{u}=0.245 \cdot \bar{u}, \beta_{w}=90^{\circ}, v=140 \mathrm{~km} / \mathrm{h}$ ).

The admittance of all other wind load components have been set to $\left|X_{K}(f)\right|^{2}:=1$ as recommended in (EN 140676: 2010, Appendix J).

Fig. 17 presents the transient wheel forces, which all start from the static wheel load $f_{w}(t=0)=57727 \mathrm{~N}$, since the wind loads are applied according to (10) with time constant $t_{0}=2 \mathrm{~s}$.

The lowest frequency that has been considered in (5) to transfer the PSD in Fig. 5 into the time domain is $f_{1}=0.01 \mathrm{~Hz}$. In the (EN 14067-6: 2010, Appendix J), it is proposed to choose the simulation time in such a way, that one full period of the lowest frequency is covered, i.e. $100 \mathrm{~s}$ here. The additional $10 \mathrm{~s}$ have been appended in order to account for the low passed filtered load application at the beginning of the simulation. Note, the (EN 140676: 2010) requests to repeat this simulation with different random phases $\phi_{i}$ in (5) and to statistically determine the mean value of the critical wind velocity and its confidence interval.

The fifth curve in Fig. 17 displays the low pass filtered wheel unloading that reaches the crosswind stability criterion at $t=90.3 \mathrm{~s}$.

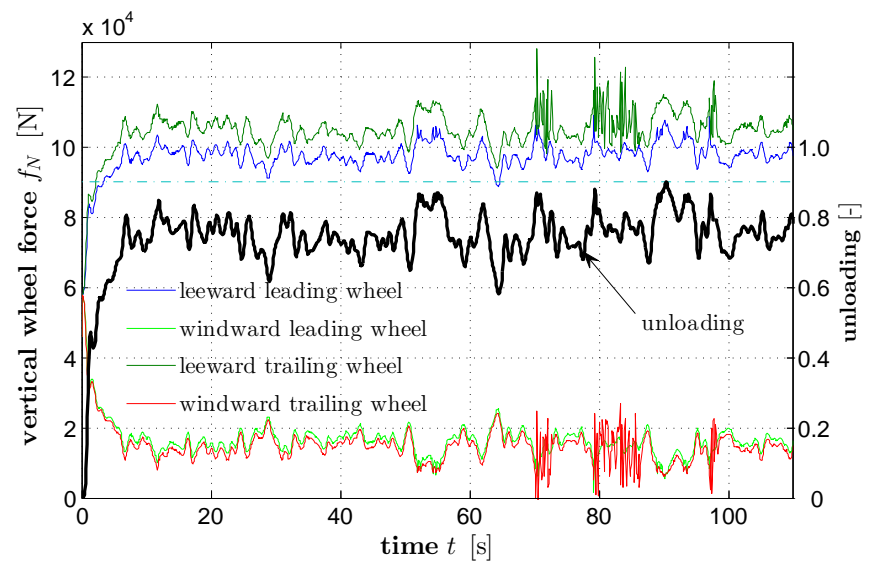

Figure 17. Transient vertical wheel force and unloading results based on hypothetical admittance parameters. 


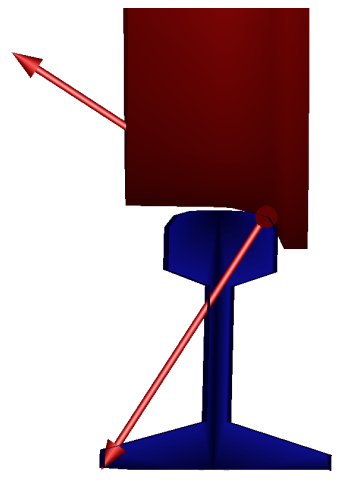

leeward train side

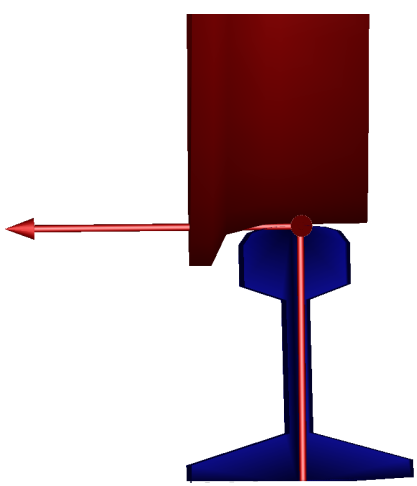

windward train side
Figure 18. Wheel-rail contact configuration with flange contact at the leeward train side

Fig. 18 illustrates the corresponding contact configuration between wheel and rail. Due the lateral wind load the complete car is displaced in lateral direction until the wheel flange is touched and counteracts the load force.

Fig. 19 shows the CWC of the NGT coach, which summarizes the critical wind velocities for 15 different vehicle speeds $\left(a_{q}=0 \mathrm{~m} / \mathrm{s}^{2}\right)$. Note, that all curves in Sec. 5.2 are preliminary results. Measured admittance functions for the NGT are not yet available, so that the associated parameters only have been chosen on a trial basis. The introduced root mean square of the wind fluctuations $\sigma_{u}=0.245 \cdot \bar{u}$ that lead to rather large peaks of the wind velocities in Fig. 16 is another parameter to be substantiated in the future.

182 cpu-s on a lap-top with Core-i7 processor and 2.9 $\mathrm{GHz}$ clock rate were required to get the above given results associated to $110 \mathrm{~s}$ simulation time. It took less than an hour to evaluate the CWC in Fig. 19, which relies on an iterative process to obtain the critical wind velocity for all 15 vehicle speeds.

\section{Conclusions and Outlook}

In the course of the DLR project Next Generation Train the subpackage CrosswindStability of the DLR Railway-

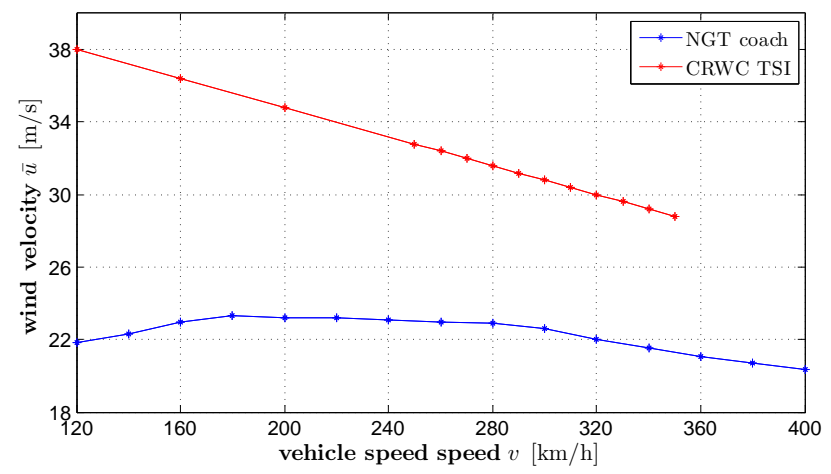

Figure 19. Preliminary critical wind curve of the NGT coach based on hypothetical admittance parameters for stochastic analysis.
Dynamics Library has been implemented. The tool offers the capability of use and combine several vehicle models and aerodynamic approaches in order to assess the crosswind stability of railway vehicles.

The consideration of unsteady aerodynamics within this task is a active field of research at DLR. The current focus is the measurement of the aerodynamic admittance function in a reproduceable and reliable manner. The RailwayDynamics Library now affords to rapidly analyze the vehicle dynamics once a aerodynamic admittance is available and that way provides a quick insight on further implications with respect to the risk of overturning.

Another future field of application of the presented capabilities concerns the dimensioning of suspension parameters of a railway vehicle in early engineering phases. Although the multibody model considers all relevant degrees of freedom and suspension components only moderate computational resources are required. The employment of the vehicle dynamics model in optimization tasks seems to be feasible, which may include multiple design objectives such as passenger comfort or running behavior besides crosswind stability.

\section{Acknowledgment}

An initial version of the Modelica crosswind stability tool has been implemented by Dr. Antonio Carrarini during his period of employment at DLR.

Wind tunnel measurements on the steady aerodynamics of the NGT have been provided by Sigfried Loose and his colleagues from the DLR Institute of Aerodynamics and Flow Technology, see Fig. 3 and cf. (Heckmann et al., 2014b).

\section{References}

M. Arnold and H. Netter. Wear profiles and the dynamical simulation of wheel-rail systems. Progress in Industrial Mathematics at ECMI, 96:77-84, 1997.

Chris Baker, Federico Cheli, Alexander Orellano, Nicolas Paradot, Carsten Proppe, and Daniele Rocchi. Cross-wind effects on road and rail vehicles. Vehicle System Dynamics, 47(8):983-1022, 2009. doi:10.1080/00423110903078794.

C.J. Baker. Ground vehicles in high cross winds part II: unsteady aerodynamic forces. Journal of fluids and structures, 5(1): 91-111, 1991.

C.J. Baker. The simulation of unsteady aerodynamic cross wind forces on trains. Journal of Wind Engineering and Industrial Aerodynamics, 98(2):88-99, 2010.

R.K. Cooper. Atmospheric turbulence with respect to moving ground vehicles. Journal of wind engineering and industrial aerodynamics, 17(2):215-238, 1984.

EN 14067-6: 2010. Railway Applications -Aerodynamics- Requirements and test procedures for crosswind assessment., 2010 . 
Uwe Fey, Johannes Haff, Mattias Jönsson, Sigfried Loose, and Claus Wagner. Experimental investigation of topological changes in the flow field around high-speed trains with respect to reynolds number scaling effects. In J. Pombo, editor, The Second International Conference on Railway Technology: Research, Development and Maintenance, number P32 in Civil Comp Proceedings, pages 1-20. Civil-Comp Press, Stirlingshire, UK, 2014. doi: 10.4203/ccp.104.32.

A. Heckmann, A. Keck, I. Kaiser, and B. Kurzeck. The Foundation of the DLR RailwayDynamics Library: the Wheel-RailContact. In 10th International Modelica Conference, 2014a.

Andreas Heckmann, Bernhard Kurzeck, Tilman Bünte, and Sigfried Loose. Considerations on active control of crosswind stability of railway vehicles. Vehicle System Dynamics, 52(6):759-775, 2014b.

Heinrich Hertz. Über die Berührung fester elastischer Körper. Journal für die reine $u$. angewandte Mathematik, 92, 1882.

S. Iwnicki. Handbook of railway vehicle dynamics. CRC Press, 2006.

B. Kurzeck, A. Heckmann, C. Wesseler, and M. Rapp. Mechatronic track guidance on disturbed track: the trade-off between actuator performance and wheel wear. Vehicle System Dynamics, 52(sup1):109-124, 2014.

Christoph Schwarz, Andreas Heckmann, and Alexander Keck. Different models of a scaled experimental running gear for the DLR RailwayDynamics Library. In 11th International Modelica Conference, 21.-23. Sep. 2015, 2015.

Mark Sterling, Chris Baker, Abdessalem Bouferrouk, Hugh ONeil, Stephen Wood, and Ewan Crosbie. An investigation of the aerodynamic admittances and aerodynamic weighting functions of trains. Journal of Wind Engineering and Industrial Aerodynamics, 97(11):512-522, 2009.

TSI HS RST 2008. 2008/232/EC: Commission Decision of 21 February 2008 concerning a technical specification for interoperability relating to the rolling stock sub-system of the trans-european high-speed rail system, 2008. 